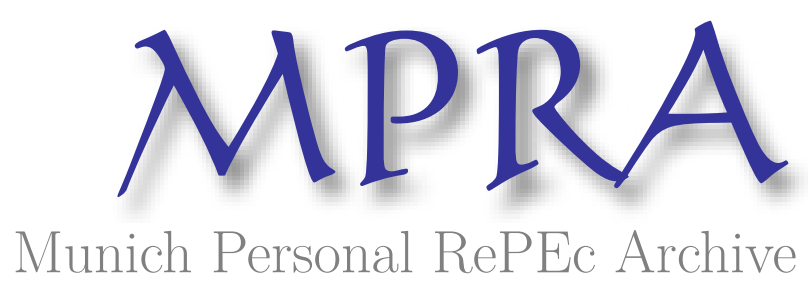

\title{
A Sequential Allocation Problem: The Asymptotic Distribution of Resources
}

Osorio, Antonio

3 June 2014

Online at https://mpra.ub.uni-muenchen.de/56690/

MPRA Paper No. 56690, posted 18 Jun 2014 23:46 UTC 


\title{
A Sequential Allocation Problem: The Asymptotic Distribution of Resources
}

\author{
António Osório ${ }^{\dagger}$ \\ ${ }^{\dagger}$ Universitat Rovira $i$ Virgili, Departament d'Economia and CREIP \\ (antonio.osoriodacosta@urv.cat)
}

\begin{abstract}
In this paper, we consider a sequential allocation problem with $n$ individuals. The first individual can consume any amount of a resource, leaving the remainder for the second individual, and so on. Motivated by the limitations associated with the cooperative or non-cooperative solutions, we propose a new approach from basic definitions of representativeness and equal treatment. The result is a unique asymptotic allocation rule for any number of individuals. We show that it satisfies a set of desirable properties.

Keywords: Sequential allocation rule, River sharing problem, Cooperative and non-cooperative games, Dictator and ultimatum games.

JEL classification: C79, D63, D74.
\end{abstract}

\section{Introduction}

We analyze the sequential allocation of a divisible resource among $n$ individuals that are ordered along a line. A well-known example of this particular situation is the stylized river sharing problem. ${ }^{1}$ The river flow is analogous to a resource or endowment and the countries, states or cities through which it passes are the individuals. The first individual (in the upstream) may

\footnotetext{
${ }^{1}$ Our problem can be viewed as a particular case of a more general river sharing problem. Since the axioms and definitions that we use in our framework may vary from those of a more general river sharing problem, it is treated as an independent problem of its own. There are also similarities with the well-known dictator game of Kahneman et al. (1986), the ultimatum game of Güth et al. (1982) or the airport problem (Littlechild and Owen, 1973), see below.
} 
consume any amount of the available resource, leaving the remaining for the second individual, and so on.

Often, a solution is enforced by third parties, but it can also be the result of negotiations between the individuals. Failures in negotiations are common, and disagreements may eventually end up in international courts. ${ }^{2}$ In this respect, the legal perspective on river sharing disputes includes the historical absolute territorial sovereignty (ATS) principle in which a country has absolute sovereignty over the flow on its territory, regardless of any harm it may cause to other downstream countries. This prior appropriation principle is compatible with non-cooperative and strictly self-interested behavior. It is widely recognized as unfair. ${ }^{3}$ Limited territorial sovereignty (LTS) has become the most important principle in international water law. Countries must respect each other's rights. The doctrine of equitable resources utilization applied to our setting includes the equal allocation as a particular case.

Since the first individual can consume the entire resource, there may not be any incentives to negotiate an agreement with the second individual. These disputes or series of negotiations are often deadlocked, i.e., no agreement can be reached. The question is: What would be a mutually agreeable solution for both parties?

In the present paper we do not assume explicitly the existence of a third party that can enforce a particular allocation. Our goal is to present a practical and realistic solution built on strong arguments to be sufficiently consensual among the involved parties in order to be naturally enforced. We search for a compromise between a (game theoretic) non-cooperative and a cooperative outcome, or from the legal perspective, a compromise between the ATS and LTS principles. Therefore, we do not excessively restrict the solution design. At the same time, we do not want to induce a particular result. We achieve it through a set of axioms that imply an admissible set of allocations. Moreover, the solution must be unique, representative and treat

\footnotetext{
${ }^{2}$ Ambec et al. (2013) address the vulnerability and monitoring difficulties associated with the compliance of existing water sharing arrangements.

${ }^{3}$ Carraro et al. (2007) and Ambec and Ehlers (2008b) survey the literature on noncooperative and cooperative solutions for the river sharing problem. Parrachino et al. (2006) review the literature.
} 
every admissible allocation equally. Some of these concepts are ambiguous. For that reason, we axiomatize them for our context.

Mathematically, we consider a discrete action space. This allows for a countable and easier computation of the sum of each individual payoff (the description of the procedure is detailed in Section 4). The sum over the individuals' payoffs gives the share of each individual on the total endowment. Asymptotically, the relative difference between allocations vanishes. The result is a unique distribution that equally weighs every admissible claim that could possibly be proposed with a continuous action space. Later, we show that it satisfies a set of desirable properties.

Related literature - Approaches based on cooperative game theory have been extensively applied to sequential allocation problems such as the river sharing problem. A study that is sufficiently representative and that has received some attention in the literature is Ambec and Sprumont (2002). They define a core lower bound and an aspiration upper bound on the welfare of a coalition of agents that uniquely determine the "downstream incremental distribution" to allocate the total welfare among the agents. ${ }^{4}$ The marginal contribution of each member of the coalition determines the individual shares. On the contrary, we do not explicitly consider monetary transfers or any other compensation or trade mechanism. For the sake of generality, we do not explicitly define a utility function. ${ }^{5}$

Ansink and Weikard (2012) treat a river sharing problem as a sequence of two-agent river sharing problems, and show a mathematical equivalence to bankruptcy problems. Their goal is the same as ours; a fair sequential distribution without monetary compensations and a unidirectional flow. However, in our setting, claims are not well defined.

Rationing problems, as in Moulin (2000), follow a sequential structure that can be adapted to our setting. Priority rules with ordered individuals, first, allocate resources to the upstream individuals until their claims are

\footnotetext{
${ }^{4}$ See Ambec and Ehlers (2008a) for an extension of downstream incremental distribution for single-peaked preferences. See also Kilgour and Dinar (2001) and Wang (2011), among others.

${ }^{5}$ Utility assumptions are subjective with profound implications on the results. Dinar et al. (1992) critique the use of game theoretical based transfers that are not related to market prices, and the representation of the problem in the "utility-space". More recently, the same position is defended in Houba (2008).
} 
satisfied. In our setting, this implies that the most upstream individual consumes the full endowment.

Herings and Predtetchinski (2012) consider a sequential bargaining protocol, in which each individual endowment share is sequentially determined. The sequential structure can be adapted to our setting. Alternating-offer bargaining models have in common the threat of delay and the equilibrium unanimity requirement. We do not impose unanimity. Instead, we search for a proposal that minimizes the potential of bargaining impasses. Moreover, in real life situations, veto power might have enforcement limitations.

Since we propose an allocation rule and an associated procedure that is new to the literature, the rest of this section is dedicated to further motivate its existence.

The non-cooperative equilibrium is unfair - In a non-cooperative context rational behavior implies that the most upstream individual consumes the full resource and passes nothing to the other individuals. The structure is similar to the well-known dictator game, Kahneman et al. (1986). Similarly, in the well-known ultimatum game (Güth et al., 1982), the downstream individual can decline the upstream individual proposal. In that case both parties obtain zero (or asymptotically zero) payoffs. In terms of our setting, this is equivalent to an impasse in the negotiation process. However, reality is not so strict, as further negotiations may take place. The equilibrium is asymptotic, which is similar to the one in the dictator game.

The theoretical predictions are a consequence of the location advantage of the upstream over the downstream individuals, and the "more is better" property of the utility function. These results are very unequal, and hard to defend. From an equity point of view, every individual must receive something. What is not clear is the value of this share. It motivates the search for this "something" but without ignoring that an upstream individual has at least a weak advantage over the subsequent individual, and so on.

Note also that the individuals that are farther downstream have less bargaining power because of their less desirable location. Consequently, the only way to protect their position is to rely on the individuals' sense of equity and justice. Empirical studies and controlled experiments in the dictator (Camerer, 2003; Engel, 2011; among others) and ultimatum (see Camerer and Thaler (1995) for a survey) games point to the existence of altruistic and equity-concerned behaviors contradicting the theory. This is also true 
for contexts like ours, in which decisions are expected to be more carefully considered (taken by groups, countries, governments, etc.), closer to rational behavior and more self-interested than individual decisions (Charness and Sutter, 2012).

The limitations of the cooperative approach - The "equity theory" of social psychology (Adams, 1963), states that each individual allocation should depend on the relation between contributions (inputs) and benefits (outcomes). In our setting this ratio is the same for all individuals. Consequently, each individual should be treated in the same way. This definition ignores strategic issues related with the position of the individuals in the sequence which is crucial in our setting. However, this aspect also limits the possibility of considering meaningful coalitions between individuals. For instance, a coalition between the second and third individual that ignores the first individual is limited because the flow passes through individual one first. Similarly, a coalition between the first and the third individual is not independent from the second individual. Therefore, some coalitions are restricted because the involved parties cannot agree on splitting something that they do not own or control ex-ante. While in theoretical terms or with further assumptions we can think on solutions to problems of this kind (see, for instance Gengenbach et al. (2010)), in practical terms they may be difficult to implement.

These difficulties motivate the search for an allocation rule outside the cooperative and non-cooperative setup. ${ }^{6}$

The paper is organized as follows. Section 2 presents the model. Section 3 defines a set of axioms that must be satisfied. Section 4 describes the procedure. Section 5 and 6 present our result and investigate its properties. Finally, Section 7 concludes with some extensions and practical issues.

\section{The Sequential Allocation Model}

Consider a divisible resource $E \in \mathbb{R}_{+}$to be allocated sequentially to a group of individuals, whose set is denoted by $N=\{1, \ldots, n\}$. Individuals are

\footnotetext{
${ }^{6}$ Nonetheless, we will show a connection between the allocation rule proposed in the present paper and the Shapley (1953) value of a particular TU game with a structure similar to an airport type problem (Littlechild and Owen, 1973; Thomson et al., 2007).
} 
identified with respect to their relative position. If $i<j$ we say that $i$ is upstream from $j$ or that $j$ is downstream from $i .{ }^{7}$ In other words, individual 1 is the first to have access to the endowment and to consume an amount $c_{1} \in[0, E]$. The remaining resource, $E-c_{1}$, is passed to individual 2, which consumes $c_{2} \in\left[0, E-c_{1}\right]$ and passes the remaining to individual 3 , and so on. The process ends with the individual $n$, which consumes the remaining endowment $c_{n}=E-\sum_{i=1}^{n-1} c_{i}$.

\section{Properties of the solution design}

We have pointed out the limitations of game theory as a tool to deal with sequential allocation problems as the one in the present paper. Moreover, we do not assume explicitly the existence of a third party that can enforce a particular allocation. Instead, we search for a compromise between the noncooperative approach, in which the full resource is consumed by the most upstream individual, and a cooperative agreement in which the resource is equally split. In other words, we do not restrict excessively the solution design to not remove the non-cooperative nature of the problem and the possibility of a potential equal division agreement. At the same time, we do not want to induce a particular result.

So far, from the discussion, we have concluded that individual $i \in N$ cannot get more than individual $i-1$ and no less than individual $i+1$; an implication of the positional disadvantage and advantage of individual $i$ with respect to individual $i-1$ and $i+1$, respectively.

Axiom 1 (Strategic Advantage). If $i<j$ then $c_{i} \geq c_{j}$ for all $i, j \in N$.

Individual $i$ knows that it is better positioned than agent $j$. A proposal that does not reflect this in terms of payoffs (at least weakly) is unacceptable from this individual's perspective. Therefore, to reach a consensual agreement among the involved parties, we must be realistic about the requirements that we impose.

Moreover, every individual, independently of its position, must receive something. This argument does not ignore equity issues and is based on the idea of fairness and justice.

\footnotetext{
${ }^{7}$ We can think of $E$ as a river flow that passes through a number of countries, regions or cities as the individuals in our context.
} 
Axiom 2 (Non-zero Payoff Right). $c_{i}>0$ for all $i \in N$.

As in the non-cooperative solution of the ultimatum game the proposer must offer some non-zero share of the total resource in order to obtain acceptance from a rational receiver. The axiom imposes that even in the worst case scenario every individual must obtain a measurable non-zero share of the total resource.

Definition 1 (Admissibility). An allocation profile that simultaneously satisfies axioms 1 and 2 is called admissible. The set of such allocation profiles is called the admissible set. ${ }^{8}$

Contrary to most of the literature on allocation problems, we do not have a utility or welfare maximizing objective. Our goal is to obtain a practical and realistic solution that can get consensus among the involved parties. If there are several of these solutions this objective is at risk, as individuals may be split between the available alternatives. The final solution must be unique, representative and treat equally every allocation proposal.

Uniqueness is a desired and well-defined property. However, representativeness and equal treatment can be subjective and object of discussion. In order to avoid this [philosophical] inconclusive debate, we objectively axiomatize their meaning to our context. ${ }^{9}$

Representativeness - Under this principle we imagine an uncountable set of solutions suggested by different individuals. Some of these proposals might be more self-interested, while others are more equity-oriented. In our perspective, axioms 1 and 2 form a sufficient representative basis for any realistic proposal.

${ }^{8}$ Axioms 1 and 2 impose the following payoff bounds, $c_{1} \in[E / n, E), c_{i} \in(0, E / i)$ for $i \in\{2, \ldots, n-1\}$, and $c_{n} \in(0, E / n]$.

The set of admissible payoff profiles that satisfy these bounds is uncountable. This aspect leads to some technical issues that are addressed later. Table 1 presents the (unit) discretized set of admissible payoff profiles for $n=3$ and $E=3,6,9,12$.

${ }^{9}$ The reader is free to consider other interpretations. There is no indisputable definition of "representativeness" and "equal treatment". "Indisputable", is also a not well-defined concept. 
Axiom 3 (Representativeness). The final allocation is representative if it receives as input every admissible allocation.

Representativeness is framed inside the set of admissible allocations. In technical terms, by representativeness each allocation in this set receives some strictly positive weight.

Equal treatment of allocations - It determines that each admissible allocation is equally important. In our setting the equal treatment is over the set of admissible allocations and not over the individuals. There is a duality between individuals and allocations. Intuitively, each allocation could have been legitimately proposed by some individual in some context. Therefore, it is equally weighted - the principle of equal treatment [over proposals].

Axiom 4 (Equal treatment of allocations). The final solution satisfies equal treatment of allocations if every allocation is uniformly weighted.

An equal treatment of allocation proposal removes from the final allocation any bias, prejudice, or individual preference that are not founded in the different strategic position of the individuals. Other distributions would have introduced other sort of bias that we cannot justify in general without an underlying theory that supports it. ${ }^{10}$

\section{The description of the procedure}

In this section, we describe in detail the construction of our allocation rule. In particular, we describe the mathematical representation of the principles presented in the previous section.

\subsection{Continuous versus discrete action space}

In a continuous action space, between two admissible allocation profiles that satisfy the bounds in footnote 8 there is an uncountable set of possible allocations. Actually, the meaning of "between" is not well defined. The

\footnotetext{
${ }^{10}$ Note the analogy between equal weighting and the Shapley (1953) value in which each individual marginal contributions to the coalition is equally weighted. The difference with respect to the Shapley value is that we do not think in terms of forming meaningful coalitions but in terms of admissible allocations. In Section 6 below we formalize the connection between these two concepts.
} 


\begin{tabular}{cccc}
\hline$E=3$ & $E=6$ & $E=9$ & $E=12$ \\
\hline $1,1,1$ & $4,1,1$ & $7,1,1$ & $10,1,1$ \\
& $3,2,1$ & $6,2,1$ & $9,2,1$ \\
& $2,2,2$ & $5,3,1$ & $8,3,1$ \\
& & $5,2,2$ & $8,2,2$ \\
& $4,4,1$ & $7,4,1$ \\
& & $4,3,2$ & $7,3,2$ \\
& & $3,3,3$ & $6,5,1$ \\
& & & $6,4,2$ \\
& & & $6,3,3$ \\
& & & $5,5,2$ \\
& & & $5,4,3$ \\
sum(total $)$ & sum(total $)$ & sum(total $)$ & sum $($ total $)$ \\
$1,1,1(3)$ & $9,5,4(18)$ & $34,18,11(63)$ & $81,40,23(144)$ \\
\hline
\end{tabular}

Table 1: The set of admissible allocation profiles for $n=3$ and $m=1,2,3,4$.

comparison between two profiles always implies that at least some individual fares better at the expense of another individual. In order to express these concepts mathematically, we consider a discrete action space because it is easier to account for all admissible allocation profiles of definition 1 . This set is now countably infinite. ${ }^{11}$ In other words, we move from the usual continuous space in which resources and allocations are values in $\mathbb{R}_{+}$, to a discrete space in which resources and allocations are values in $\mathbb{N}_{1}$. Finally, we obtain an asymptotic distribution that is valid in the former space. The discretized set of admissible allocations for $n=3$ and $E=3,6,9,12$ is given in Table 1.

\subsection{The construction of the procedure}

We start by considering the following example. Suppose that $n=3$ and $E=3$. In a discrete decision space case there is one admissible allocation that simultaneously satisfies axioms 1 and 2 , i.e., $(1,1,1)$. The allocations $(2,1,0)$ and $(3,0,0)$ fail the non-zero requirement of axiom 2 . However, if

\footnotetext{
${ }^{11}$ The need of a discrete action space is also motivated by the problem of defining arbitrarily small or large values on a real number system.
} 
$E=6$ there are three admissible allocations that simultaneously satisfies axioms 1 and 2, i.e., $(4,1,1),(3,2,1)$ and $(2,2,2)$, see Table 1 .

This process of generating admissible allocations can be generalized by letting $E=n m$, where $m=1,2, \ldots$, and $n$ is the number of individuals (see Remark 1 in the end of this section for a technical explanation). ${ }^{12}$

Subsequently, for a given $n$ and $m$, we sum vertically the individual $i \in N$ payoffs and denote this sum as $\operatorname{sum}_{i}^{n}(m)$. At the bottom of Table 1, we show each individual sum of payoffs over all admissible allocations. Every admissible allocation contributes equally for the final allocation, i.e., the principle of representativeness and equal treatment, axiom 3 and 4, respectively. The objective is to find a general expression or recursion that characterizes the sum of the values in the sequence for any $m$.

In the next step we define the ratio of the individual $i$ sum of admissible payoffs, with respect to the total, i.e.,

$$
r_{i}^{n}(m) \equiv \operatorname{sum}_{i}^{n}(m) / \sum_{k=1}^{n} \operatorname{sum}_{k}^{n}(m) .
$$

The result is the share of individual $i$ on the total resources. Finally, we consider the limit of $r_{i}^{n}(m)$ for $m \uparrow \infty$.

For instance, in Table 1 the individual 1 ratio grows from $r_{1}^{3}(1)=1 / 3$ for $m=1$ to $r_{1}^{3}(4)=81 / 144$ for $m=4$. For $m \uparrow \infty$, the individual 1 ratio converges to $r_{1}^{3} \equiv r_{1}^{3}(\infty)=11 / 18$ (see Proposition 1 below). In other words, our allocation rules states that the individual located farthest upstream must receive $61.1(1) \%$ of the total resource under dispute. Formally,

Definition 2. The individual $i \in N$ asymptotic allocation is defined as

$$
\phi_{i}^{n} \equiv r_{i}^{n} E \equiv \lim _{m \rightarrow \infty} \frac{\operatorname{sum}_{i}^{n}(m)}{\sum_{k=1}^{n} \operatorname{sum}_{k}^{n}(m)} E
$$

where $r_{i}^{n}$ represents the asymptotic share of the total resource.

Note the compromise between cooperative (preferred by the individuals located the farthest downstream) and non-cooperative behavior (preferred by the individuals located the farthest upstream). For instance, if $E=9$ (see Table 1), we are considering admissible allocations that can be regarded

\footnotetext{
${ }^{12}$ Following the discussion, with a discrete action space, we can rewrite the bounds in footnote 8 as $c_{1} \in[m, n m-1]$, and $c_{i} \in[1, n m / i]$, for $i \in N \backslash 1$.
} 
as the result of a more cooperative motivated agreements, i.e., $(3,3,3)$ or $(4,3,2)$, and admissible allocations that seem to be the result of a more non-cooperative motivated agreement, i.e., $(7,1,1)$ or $(6,2,1)$. In between, there are admissible allocations that may not fall in any of these two extreme cases, i.e., $(5,3,1),(5,2,2)$ and $(4,4,1)$.

Note that as $m \uparrow \infty$ (or equivalently, as $E \uparrow \infty$ ), the discretization becomes finer and vanishes. Therefore, we consider every admissible allocation profile that could possibly be built with a continuous action space.

Recall that we started the discussion justifying the passage from a continuous to a discrete consumption space. Now, asymptotically, we move back from the discrete to the continuous space to obtain a unique distribution.

Remark 1. We consider $E=n, 2 n, \ldots, m n, \ldots,($ for $E<n$ the admissible set is empty) instead of $E=1,2, \ldots, k, \ldots$. Asymptotically, for $m \uparrow \infty$ or $k \uparrow \infty$, both approaches are equivalent. The chosen approach simplifies the computation of the general expressions that characterize the sum of each individual payoff as a function of $m$.

\section{The Allocation Rule}

Our ultimate objective is to obtain a general expression for the total resource asymptotic share $\phi_{i}^{n}$ of individual in position $i \in N$ as a function of the total number of individuals $n$.

Proposition 1. The admissible asymptotic allocation is,

$$
\phi_{i}^{n}=\frac{1}{n} E \sum_{k=i}^{n} \frac{1}{k}
$$

for $i=1, \ldots, n$ and $n=1,2, \ldots$

The contribution and equal weight of every allocation that can be defended as a possible final agreement (admissible allocation) are some strong points of our allocation rule. The obtained solution is also unique and analytical which is of great relevance for applied work.

In terms of intuition, each term in the summation $\sum_{k=i}^{n} 1 / k$ in expression (2) can be seen as representing the value of being in the position $k$ for individual $i$. The summation in expression (2), that defines the allocation 
$\phi_{i}^{n}$, is then the sum of all feasible values. For instance, in a $n=3$ problem, the individual $i=1$ can be located first, second or third, with respective values, $1,1 / 2$ and $1 / 3$. However, the individual $i=2$ cannot be located first because individual $i=1$ would not allow it. Consequently, the individual $i=2$ can be located second or third, with respective values, $1 / 2$ and $1 / 3$. Finally, individual $i=3$ has no other possibility than being always in the third position which has a value of $1 / 3$.

There is also an intuitive relation between the allocation proposed in (2) and the Shapley (1953) value of a particular TU game. This issue is discussed in the following Section.

\section{Some comments on empirics}

Contrary to most of the literature in allocation problems that focus on welfare or other maximizing objectives, we have a general agreement and easy application objective. Therefore, if the proposed allocation rule replicates average or representative human behavior, even in very challenging and subtle situations, then it is more likely that our consensus objective is reached. In this respect, our results coincide with some controlled experiments and empirical studies.

For instance, in the case $n=2$ our admissible asymptotic allocation is,

$$
\phi^{2}=(3 / 4,1 / 4) E .
$$

Engel (2011) aggregates information of 129 published papers on the dictator game and found that dictators on average keep a share of $71.65 \%$, which is close to the $75 \%$ proposed by our allocation rule. Other papers predict similar values depending on the treatment.

In the case $n=3$ our admissible asymptotic allocation is,

$$
\phi^{3}=(11 / 18,5 / 18,2 / 18) E .
$$

Bahr and Requate (2014) perform an experimental design with a structure similar to our sequential allocation problem. They found that when the share of the first individual is in between $50-66 \%$, the share of the second individual is between $21-31 \%$. Our allocation rule suggests approximately $61 \%$ and $28 \%$, respectively. Similarly, Bonein et al. (2007) performed experiments in a sequential dictator game. In one treatment, the individuals 2 and 3 played an ultimatum game. In the other treatment, they played a dictator game. The 
offer of individual 2, as a percentage of the offer of individual 1, was around $40 \%$, in the former and around $30 \%$, in the latter case. Our allocation rule predicts $40 \%$ of the individual 1 offer.

\section{Properties of the Allocation Rule}

In this section, we show that the proposed solution satisfies a set of desirable properties.
P1: (monotonic decreasing allocation share with $i$ ) $\phi_{i}^{n}>\phi_{i+1}^{n}$, for $i=1,2, \ldots, n-1$.

It is the most natural property. The higher the individual in the stream, the larger is its share on the total resource. This property is connected with the strategic advantage principle of axiom 1.

P2: (monotonic decreasing allocation share with $n$ ) $\phi_{1}^{n}>\phi_{1}^{n+1}$ and $\phi_{i}^{n}>\phi_{i+1}^{n+1}$, for $i=1,2, \ldots, n$.

The first part, i.e., $\phi_{1}^{n}>\phi_{1}^{n+1}$, states that the allocation to the most upstream individual, $i=1$, decreases as the number of individuals increases. This property should be natural for every individual allocation. However, for the other downstream individuals there is some ambiguity in the comparisons. The addition of one or more new individuals may change the ordering which has strategic implications. For instance, consider the individual $i=3$ in $n=3$. If we add a new individual $i_{\text {new }}$ the allocation share of individual $i$ is going to depends crucially on the position that this new individual will occupy in the sequence. If this new individual is positioned at $i_{\text {new }} \leq 3$ then individual $i$ is pushed to the fourth position (i.e., it becomes $i=4$ ) and consequently obtains a lower allocation share. This is what the second part (i.e., $\left.\phi_{i}^{n}>\phi_{i+1}^{n+1}\right)$ of property $P 2$ says; there exists a monotonic decreasing relation with $n$ in the downward diagonal. Table 2 provides a numerical illustration. Otherwise, if the new individual is positioned at $i_{\text {new }}=4$, then the individual $i=3$ may become better-off (which is not true in general).

This property should not be confused with the common monotonicity property in "bankruptcy" or other resource sharing type problems, in which no individual can get better-off when the same resource is divided among a larger number of individuals. In a sequential setting any conclusion depends crucially on the position that the new individual will occupy in the sequence. 


\begin{tabular}{lccccc}
\hline & $n=2$ & $n=3$ & $n=4$ & $n=5$ & $n=6$ \\
\hline$i=1$ & 0.75000 & $0.6111 \overline{1}$ & $0.5208 \overline{3}$ & $0.4566 \overline{6}$ & $0.4083 \overline{3}$ \\
$i=2$ & 0.25000 & $0.2777 \overline{\overline{7}}$ & $0.2708 \overline{3}$ & $0.2566 \overline{\overline{6}}$ & $0.2416 \overline{\overline{6}}$ \\
$i=3$ & & $0.1111 \overline{1}$ & $0.1458 \overline{3}$ & $0.1566 \overline{6}$ & $0.1583 \overline{3}$ \\
$i=4$ & & & 0.06250 & 0.09000 & $0.1027 \overline{\overline{7}}$ \\
$i=5$ & & & & 0.04000 & $0.0611 \overline{1}$ \\
$i=6$ & & & & & $0.0277 \overline{7}$ \\
\hline
\end{tabular}

Table 2: Individual asymptotic shares of the total endowment for $n=2,3,4,5,6$.

Therefore, careful should be taken when transposing properties standard in static to sequential settings. Not always exist an immediate analogous between these two worlds because of the different strategic considerations.

\section{P3: (monotonic decreasing relative share with $i) \phi_{i}^{n} / \phi_{i+1}^{n}>\phi_{i+1}^{n} / \phi_{i+2}^{n}$, for $i=1,2, \ldots, n-3$.}

The result states that the individual $i$ allocation is not only larger than that of individual $i+1$ (see property $P 1$ ) but it is also larger in relative terms than the one that $i+1$ obtains with respect to $i+2$. While in absolute terms the individuals' allocations decrease as we move downstream, in relative terms they decrease less for the most downstream individuals. In other words, as we move from $i=1$ to $i=n$, the allocation share decreases in a convex fashion. This property favors equity and it is related with property P6 below. ${ }^{13}$

\section{P4: (individual $n$ weak relative share) $\phi_{n-2}^{n} / \phi_{n-1}^{n}<\phi_{n-1}^{n} / \phi_{n}^{n}$.}

The monotonic relation of $P 3$ is not true for the last individual comparison. The relative share of individual $i=n-2$ over $i=n-1$ is lower than the relative share of individual $i=n-1$ over $i=n$. This is because individual $i=n$ is the last in the sequence, therefore, in a weak strategic position. There are two factors that play a role in each individual allocation. The first is the equity concerns of the other individuals. The second is the

\footnotetext{
${ }^{13}$ Properties $P 1, P 2$ and $P 3$ receive empirically support in Bahr and Requate (2014), Bonein et al. (2007) and Engel (2011). Engel (2011) shows that if exist more than one recipient the dictator naturally accepts a lower allocation for himself.
} 
strategic position of each individual with respect to the next one. The last individual in a sequential allocation problem does not benefit from the latter effect. Virtually, it has no strategic influence. For that reason, its allocation falls relative to the allocation of individual $i=n-1$. $^{14}$

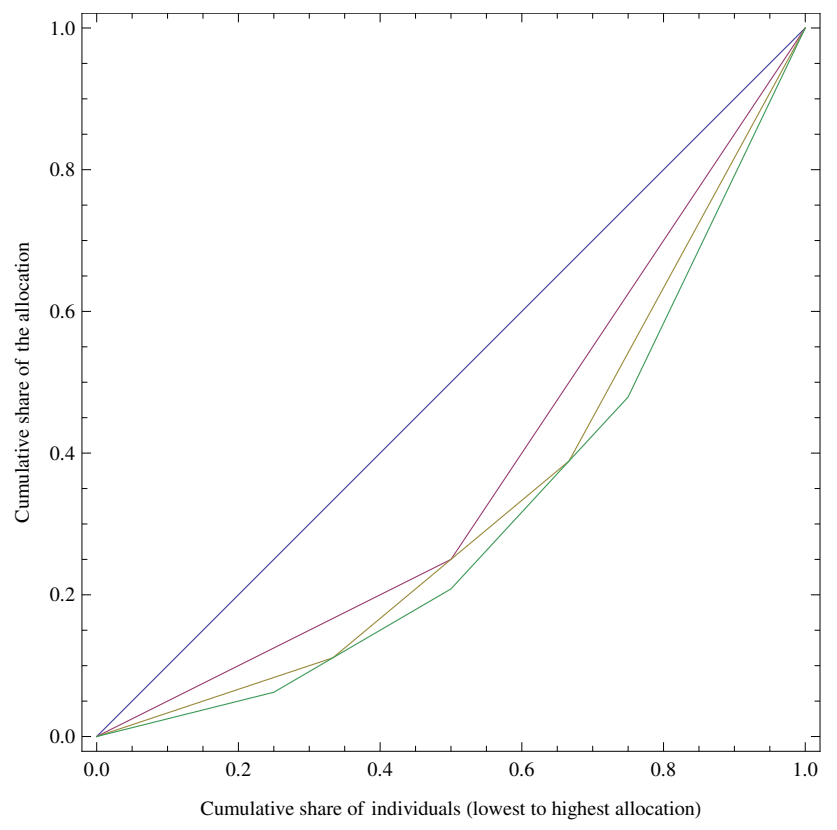

Figure 1: Lorenz curve. perfect equality curve (blue), $n=2$ (red), $n=3$ (brown), $n=4$ (green).

\section{P5: (monotonic decreasing relative share with $n$ ) $\phi_{i}^{n} / \phi_{i+1}^{n}>\phi_{i}^{n+1} / \phi_{i+1}^{n+1}$, for $i=1,2, \ldots, n-1$.}

Similar to property $P 3$, as the number of involved individuals increases, the relative share, measured by the ratio between allocations decreases, in particular for the most upstream individuals.

\section{P6: (Lorenz inequality increases with $n$ )}

\footnotetext{
${ }^{14}$ The equity concerns of the other individuals toward individual $i=1$ is negative and equals to $-\left(1-\phi_{1}^{n}\right)$, while the strategic positioning value is maximal and equal to 1 . On the other hand, the strategic positioning value of individual $n$ is minimal and equals to 0 , but benefits from positive equity concerns, which has a value of $\phi_{n}^{n}$. The distinction between equity concerns and strategy positioning value should be object of further research.
} 
Figure 1 illustrates this property. As the allocation shares are being adjusted for the increasing number of individuals and everybody tends to obtains less, see property $P 2$, it follows that the more upstream individuals' concessions to the more downstream ones decreases in relative terms. In other words, the most upstream individuals accept a reduction of their share when the number of individuals increase but this concession decreases in relative terms. Consequently, we observe an increase in inequality in Lorenz terms.

We must also note the directional interpretation of this property. For instance, if instead we consider a decrease in the number of individuals then we observe a decrease in Lorenz inequality which would be accepted as positive. Consequently, what the property is actually stating is that it is more difficult to obtain equitable agreements (in Lorenz sense) in larger than in smaller groups.

Finally, we acknowledge the importance of equity. However, it may be a utopic objective. If we constraint the allocation design on this objective, ignoring the positional advantage of the most upstream individuals, then we would have failed with the objective of presenting a practical and realistic solution to be accepted by the involved parties.

\section{P7: (Lorenz geometry)}

The edges of the $n$-Lorenz curve are tangent to the $n$-1-Lorenz curve, and so on. Figure 1 illustrates this property. Under some conditions, it is an alternative method of obtaining expression (2).

P8: (Shapley value from equal division): The admissible asymptotic allocation is the Shapley value of a particular TU game.

This property establishes the connection between the allocation proposed in (2) and the Shapley (1953) value of a particular TU game with a structure similar to an ordered cost allocation problem, e.g., the airport problem (Littlechild and Owen, 1973; Thomson et al., 2007). ${ }^{15}$ As an illustration of such coalition game, suppose that the allocation $(E / 3, E / 3, E / 3)$ is temporally agreed upon between $n=3$ individuals ordered sequentially. Then, we can

\footnotetext{
${ }^{15}$ We are thankful to an anonymous referee for pointing out this property and the description of the associated TU game.
} 
consider what each coalition can achieve by deviating from this agreement. Individual 1 on the upstream can deviate and obtain the full resource, i.e., $v(\{1\})=E$. However, if individual 1 follows the agreement and gets $E / 3$, individual 2 can deviate to obtain the remaining resource, i.e., $v(\{2\})=2 E / 3$. On the other hand, the individual 3 cannot get more than $v(\{3\})=E / 3$ which only happens if all the other individuals follow the agreement. Similarly, the coalition between individual 1 and 2 can get the full resource, i.e., $v(\{1,2\})=E$. The same happens to the coalition between individual 1 and 3. However, the coalition between individual 2 and 3 can get at most $v(\{2,3\})=2 E / 3$ if individual 1 follows the initial equal split agreement. The grand coalition formed by all individuals worth $v(\{1,2,3\})=E$. It is easy to show that for the coalition game just described the Shapley value coincides with the allocation proposed in expression (2), see Table 2. This construction can be generalized for arbitrary $n .^{16}$

In this sense, the proposed allocation can be called the "Shapley value from equal division" in just the same way as the "Walrasian allocation from equal division" (Thomson and Varian, 1985). This property is relevant because it links our seemingly unrelated approach and the Shapley value resulting from a particular airport problem. Intuitively, our approach averages over the set of admissible allocations while the Shapley value averages over the marginal contributions of individual players across all different orderings of coalition formation, see Footnote 10 above. Moreover, the observed relation suggests that for each variation of our original model (see Section 7) it may exists a particular TU game for which the Shapley value coincides with the solution obtained through our approach.

\section{Consistency and other comments}

We have presented and discussed a set of properties associated with the allocation rule proposed in (2). We now consider consistency (Thomson, 2011; Moulin, 2000; among others). In our sequential setting an equal relative treatment among neighbor individuals seems to be a natural consistency requirement.

\footnotetext{
${ }^{16}$ In spite that the Shapley value and our approach agree for the described game, there is no immediate link between the marginal contribution of each different sized coalition with each term in the summation $\sum_{k=i}^{n} 1 / k$ of expression (2) in a general and meaningful way.
} 
We motivate consistency in relative terms as follows. Suppose that $n=3$, and note that individual 1 keeps the amount $\phi_{1}^{3}$ of the available resource $E$ and passes the remaining to individual 2, subsequently, individual 2 keeps the amount $\phi_{2}^{3}$ of the available resource $E-\phi_{1}^{3}$ and passes the remaining to individual 3. In our setting, consistency means that in relative terms, individual 3 should be treated in the same way by individual 2 as individual 2 is treated by individual 1 . In other words, it imposes that $\phi_{1}^{3} / \phi_{2}^{3}=\phi_{2}^{3} / \phi_{3}^{3}$. Simultaneously, the allocation must be efficient, i.e., $\phi_{1}^{3}+\phi_{2}^{3}+\phi_{3}^{3}=E$.

Definition 3 (relative consistency). An allocation rule is consistent in relative terms if $\phi_{i}^{n} / \phi_{i+1}^{n}=\phi_{i+1}^{n} / \phi_{i+2}^{n}$ and $\sum_{i=1}^{n} \phi_{i}^{n} E=E$ for $i=1,2, \ldots, n-2$.

As defined, consistency is related with property $P 3$ above. Consequently, the allocation rule proposed in (2) does not satisfy this definition of consistency. Other interpretations of consistency are possible.

Herings and Predtetchinski (2012) propose an allocation rule that applied in our setting is given by:

$$
\phi_{i}^{n}=\delta^{i-1} / \sum_{k=1}^{n} \delta^{k-1}
$$

where $\delta \in(0,1)$ is a common discount factor. We can show that their sharing rule does not satisfy properties $P 3, P 4$ and $P 5$. The relative bargaining power is constant for varying $i$ and $n$. An implication is that, on the contrary to our allocation proposal their rule satisfies our definition of consistency.

However, we note that in sequential allocation problems of the kind presented in the current paper, consistency imposes that pairwise allocations must be linked in a predetermined (linear) order, which is mathematically convenient in some class of allocation problems, as for instance bankruptcy problems, see Thomson (2003). Moreover, there is not a unique definition of consistency.

In our setting relative consistency fails because different individuals have different strategic positions and, consequently, are affected differently. Nonetheless, the induced nonlinear effect slows down inequality which has positive equity implications and justifies the failure of consistency as it is defined above. 


\section{Extensions}

This paper is the first step in a new class of allocation rules for sequential problems. We have presented the approach that we consider to be the most focal. However, our theory is particularly flexible, the reader is free to redefine the admissible set or to reinterpret the concepts in the axioms 3 and 4, see Footnote 9 .

We now consider possible extensions associated with relaxations of axioms 1 and 2, used to define the admissible set of Definition 1. Other extensions associated with variations of the original sequential allocation problem (nonconstant resources, unequal weights, asymmetric individuals, satiation levels, etc.) are also possible.

1) One possibility is to keep axiom 2, but replace axiom 1 by its strict version. In other words, an individual located upstream must have a strict advantage over an individual located downstream.

Axiom 5 (Strict Strategic Advantage). If $i<j$ then $c_{i}>c_{j}$ for all $i, j \in N$.

2) The reverse possibility is to maintain axiom 1 but replace axiom 2 by its relaxed version. In this case, allocation profiles in which one or more individuals obtain a zero payoff are possible.

Axiom 6 (Non-Strict Payoff Right). $c_{i} \geq 0$ for all $i \in N$.

3) We can also consider the strict version of axiom 1 and the relaxed version of axiom 2 simultaneously, i.e., replace these by axioms 5 and 6 , respectively.

In the three cases considered, the distribution of the total resource tends to favor the individuals located upstream with respect to the individuals located downstream. In other words, it will necessarily result in allocations 
that are less equitable in Lorenz sense. ${ }^{17,18}$ In order to see it, consider the following example.

Example 1. Let $n=3$ and $m=2$. Under axioms 1 and 2 the admissible set is: $(4,1,1),(3,2,1)$ and $(2,2,2)$. The respective vector of individual shares is: $r^{3}(2)=\frac{1}{18}(9,5,4)$. In case 1) the admissible set is composed by a single profile, i.e., $(3,2,1)$. The respective vector of individual shares is: $r^{3}(2)=\frac{1}{18}(9,6,3)$. In case 2) the admissible set is: $(6,0,0),(5,1,0)$, $(4,2,0),(4,1,1),(3,3,0),(3,2,1)$ and $(2,2,2)$. The respective vector of individual shares is: $r^{3}(2)=\frac{1}{18}(11.6,4.7,1.7)$. In case 3) the admissible set is: $(5,1,0),(4,2,0)$ and $(3,2,1)$. The respective vector of individual shares is: $r^{3}(2)=\frac{1}{18}(12,5,1)$.

From the example, it is clear that the individual 3 share of the total resource [in cases 1), 2) and 3)] is always smaller with respect to the admissible set defined in Section 3, see Table 1. The opposite conclusion holds for individual 1, which never gets into a worse situation. Mixed results are observed for individual 2 (see the discussion after property $P 2$ ). These conclusions remain valid for larger values of $m$, in particular for $m \uparrow \infty$.

\section{A Note for Practitioners}

Some situations may justify that prior to the distribution of the total resource among the involved parties; every individual receives a minimum

\footnotetext{
${ }^{17}$ In the case $n=2$ the asymptotic distribution remains the same as in 3 . For isntance, if axiom 1 is replaced by axiom 5 , we simply remove the payoff profile $(m, m)$ from the admissible set, which appears only once for any $m$. If axiom 2 is replaced by Axiom 6 , we add the payoff profile $(2 m, 0)$. Asymptotically, a single term is irrelevant. However, for $n \geq 3$ we must have different asymptotic distributions, because the removed and/or added allocation profiles increase with $m$.

${ }^{18}$ Another possible extension is to verify the relation between the asymptotic approach of the current paper with physics concepts such as the centroid, center of mass or gravity. Note that each admissible allocation is uniformly weighted (axiom 4) but the distribution of mass is not homogeneous because the allocations are heterogeneous. Therefore, it is likely that our result is equivalent to the center of mass of the admissible set. Such a result requires knowledge about the mass density function. However, care should be taken, the relaxation of an axiom (a simple inequality) leads to a different admissible set, which is may not be captured by an integration-based method. We are thankful to an anonymous referee who pointed out the possibility of this property.
} 
amount. For instance, in a river sharing problem, observations of this kind make sense when a minimum flow is required to keep the habitat of certain species protected. Therefore, from the total river flow, only a part of it can be used for consumption. The asymptotic allocation of Proposition 1 can be straightforwardly applied to these situations in which a part of the resource is equally split or distributed according to other procedures. When justified, this kind of procedure may allow distributions that are less asymmetric and more equitable.

Acknowledgements. We would like to thank Sebastian Cano, José-Manuel GiménezGómez, Susan Glazer, Wesley Hurt, Juan Pablo Ríncon-Zapatero, Neil Sloane and Yves Sprumont for their very useful comments. The usual caveat applies. Financial support from Universitat Rovira i Virgili, Ministerio de Ciencia e Innovación under project ECO2011-24200 and the Barcelona GSE is gratefully acknowledged.

\section{Appendix}

\section{Proof of Proposition 1.}

The strategy of the proof is the following. We start by constructing the general expression associated with a given sequence of numbers to obtain the respective asymptotic ratio for $n=2,3,4$. Finally, using the obtained information we construct an algorithm that delivers the asymptotic allocation for any $i$ and $n$.

Consider $n=2$ and let the resource be $E=2 m$ for each $m=1,2, \ldots$, and $i=1,2$. We sum all admissible payoffs until a pattern emerges for each individual sum. The sequence $2,8,18,32,50,72, \ldots$, that represents the aggregate sum of payoffs over all agents and profiles has the general expression $2 m^{2}$. The sequence $1,5,12,22,35 \ldots$, that represents the individual 1 sum of payoffs over all profiles has the general expression $\operatorname{sum}_{1}^{2}(m)=$ $m(3 m-1) / 2$. Therefore, by $(1)$ the asymptotic share of the total resource is,

$$
r_{1}^{2}(m)=m(3 m-1) / 2\left(2 m^{2}\right) \rightarrow 3 / 4 .
$$

The sequence $1,3,6,10,15, \ldots$, that represents the individual 2 sum of payoffs over all profiles has the general expression $\operatorname{sum}_{2}^{2}(m)=m(m+1) / 2$. Similarly, the asymptotic fraction of the total endowment is $r_{2}^{2}(m) \rightarrow 1 / 4$.

Now consider $n=3$ and let the resource be $E=3 m$ for each $m=1,2, \ldots$, and $i=1,2$. We proceed until a pattern emerges for each individual sum. 
The set of admissible allocations for $n=3$ and $m=1,2,3,4$, is given in Table 1. The aggregate sum of payoff profiles for each individual is shown in the last row. The general expression for the sequence $3,18,63,144,285, \ldots,{ }^{19}$ that represents the aggregate sum of payoffs over all agents and profiles is

$$
\operatorname{sum}^{3}(m)=3 m\left(m^{2}-\left\lfloor m^{2} / 4\right\rfloor\right)=\left(18 m^{3}+3 m\left(1-(-1)^{m}\right)\right) / 8,
$$

where $\lfloor$.$\rfloor denotes the floor function. The expression for the sequence 1, 9,$ $24,81,163,282, \ldots$, that represents the individual 1 sum of payoffs over all profiles is

$$
\begin{aligned}
\operatorname{sum}_{1}^{3}(3, m)= & 3 m\left(m^{2}-\left\lfloor m^{2} / 4\right\rfloor\right)-\sum_{k=1}^{m}\left(2 k^{2}-\left\lfloor k^{2} / 4\right\rfloor\right) \\
& -\sum_{k=1}^{\lfloor(m-1) / 2\rfloor}(m+k)(m-2 k) \\
= & \left(22 m^{3}-6 m^{2}-(m+1)-(3 m-1)(-1)^{m}\right) / 16 .
\end{aligned}
$$

Therefore, by (1) the asymptotic fraction of the total resource is

$$
r_{1}^{3}(m)=\frac{\left(22 m^{3}-6 m^{2}-(m+1)-(3 m-1)(-1)^{m}\right) / 16}{\left(18 m^{3}+3 m\left(1-(-1)^{m}\right)\right) / 8} \rightarrow \frac{11}{18} .
$$

The expression for the sequence $1,5,18,40,80,135, \ldots$, that represents the individual 2 sum of payoffs over all profiles is

$$
\begin{aligned}
\operatorname{sum}_{2}^{3}(m) & =\sum_{k=1}^{m} k^{2}+\sum_{k=1}^{\lfloor(m-1) / 2\rfloor}(m+k)(m-2 k) \\
& =\left(10 m^{3}+3 m\left(1-(-1)^{m}\right)\right) / 16,
\end{aligned}
$$

and the asymptotic fraction of the total resource is

$$
r_{2}^{3}(m)=\frac{\left(10 m^{3}+3 m\left(1-(-1)^{m}\right)\right) / 16}{\left(18 m^{3}+3 m\left(1-(-1)^{m}\right)\right) / 8} \rightarrow \frac{5}{18} .
$$

Similarly, the expression for the individual 3 sum sequence 1, 4, 11, 23, 42, $69, \ldots$, is given by

$$
\operatorname{sum}_{3}^{3}(m)=\sum_{k=1}^{m}\left(k^{2}-\left\lfloor k^{2} / 4\right\rfloor\right)=\left(4 m^{3}+6 n^{2}+4 m+\left(1-(-1)^{m}\right)\right) / 16,
$$

\footnotetext{
${ }^{19}$ The sequences in this paper have been found by the authors and are registered in the "OEIS Foundation Inc. (2011), The On-Line Encyclopedia of Integer Sequences, http://oeis.org."
} 
and the asymptotic fraction of the total resource is $r_{3}^{3}(m) \rightarrow 2 / 18$.

Now consider $n=4$ and let the resource be $E=4 \mathrm{~m}$. We proceed for $m=$ $1,2, \ldots$, until a pattern emerges. This case is more complex, the expressions $\operatorname{sum}_{i}^{4}(m)$ for $i=1,2,3,4$, are given by non-trivial recursions. The general expression for the sequence $4,40,180,544,1280,2592, \ldots$, that represents the aggregate sum of payoffs over all agents and profiles is given by the recursion,

$$
\begin{aligned}
\operatorname{sum}^{4}[m]= & \frac{m}{m-1} \operatorname{sum}^{4}[m-1] \\
& +4 m \sum_{k=0}^{2 m}\left(\left\lfloor\frac{4 m-2-k}{2}\right\rfloor-k\right)\left\lfloor\frac{\operatorname{sgn}\left(\left\lfloor\frac{4 m-2-k}{2}\right\rfloor-k\right)+2}{2}\right\rfloor,
\end{aligned}
$$

where $\operatorname{sum}^{4}[m]=\sum_{i=1}^{4} \operatorname{sum}_{i}^{4}[m], \operatorname{sum}^{4}[1]=4$ and $\operatorname{sgn}$ denotes the sign function. The total number of admissible profiles is $s u m^{4}[\mathrm{~m}] / \mathrm{nm}$. The expression $\operatorname{sum}_{1}^{4}(m)$ for the individual 1 sum sequence 1, 17, 84, 262, 629, $1289, \ldots$, is given by the recursion,

$$
\begin{aligned}
\operatorname{sum}_{1}^{4}[m]= & \operatorname{sum}_{1}^{4}[m-1]+\frac{\operatorname{sum}^{4}[m-1]}{4 m-4} \\
& +\sum_{k=0}^{2 m} \sum_{l=k+1}^{\left\lfloor\frac{4 m-2-k}{2}\right\rfloor}(4 m-2-l-k)\left\lfloor\frac{\operatorname{sgn}\left(\left\lfloor\frac{4 m-2-k}{2}\right\rfloor-k\right)+2}{2}\right\rfloor,
\end{aligned}
$$

where $\operatorname{sum}_{1}^{4}[1]=1$ and $\operatorname{sum}^{4}[m-1]$ is given by (.1). The asymptotic fraction of the total resource is obtained numerically and is given by,

$$
r_{1}^{4}(m)=\frac{\operatorname{sum}_{1}^{4}(m)}{\operatorname{sum}^{4}(m)} \rightarrow \frac{25}{48} .
$$

The expression $\operatorname{sum}_{2}^{4}(m)$ for the individual 2 sequence 1, 10, 46, 141, 334, $680, \ldots$, is given by the recursion,

$$
\begin{aligned}
\operatorname{sum}_{2}^{4}[m]= & \operatorname{sum}_{2}^{4}[m-1]+\frac{\operatorname{sum}^{4}[m-1]}{4 m-4} \\
& \left.+\sum_{k=0}^{2 m} \sum_{l=k+1}^{\left\lfloor\frac{4 m-2-k}{2}\right\rfloor} l \frac{\operatorname{sgn}\left(\left\lfloor\frac{4 m-2-k}{2}\right\rfloor-k\right)+2}{2}\right\rfloor,
\end{aligned}
$$

where $\operatorname{sum}_{2}^{4}[1]=1$ and $\operatorname{sum}^{4}[m-1]$ is given by (.1). The asymptotic fraction of the total resource is obtained numerically and is

$$
r_{2}^{4}(m)=\operatorname{sum}_{2}^{4}(m) / \operatorname{sum}^{4}(m) \rightarrow 13 / 48 .
$$


For simplicity, we consider the individual 4 expression $\operatorname{sum}_{4}^{4}(m)$ for the sum sequence $1,6,21,55,119,227, \ldots$, which is given by the following recursion,

$$
\operatorname{sum}_{4}^{4}[m]=\operatorname{sum}_{4}^{4}[m-1]+\operatorname{sum}^{4}[m] /(4 m),
$$

where $\operatorname{sum}_{4}^{4}[1]=1$ and $\operatorname{sum}^{4}[m-1]$ is given by $(.1)$. Note that $\operatorname{sum}_{4}^{4}(m)=$ $\sum_{k=1}^{m} \operatorname{sum}^{4}[k] / k$. The asymptotic fraction of the total resource is obtained numerically and is $r_{4}^{4}(m) \rightarrow 3 / 48$. Finally, the expression $\operatorname{sum}_{2}^{4}(m)$ for the individual 3 sum sequence 1, 7, 29, 86, 198, 396,.., and the asymptotic fraction of the total resource are obtained as the residual difference. In resume, the obtained asymptotic allocation is,

$$
\phi^{4}=(25 / 48,13 / 48,7 / 48,3 / 48) E .
$$

The construction of recursions $\operatorname{sum}_{i}^{n}(m)$ for general $n$ are impractical. In spite of this, using the information obtained so far we are able to derive a general expression for each individual $i \in N$ asymptotic allocation. The argument is based in the following general algorithm that reproduces the numbers in (3), (4) and (.2), and consequently for any $n$. For the case $n=2$, we have $c_{1} \in[E / 2, E)$ and $c_{2} \in(0, E / 2]$, and by efficiency $c_{1}+c_{2}=E$. Since asymptotically we consider every admissible allocation in a continuous space we can think as if $1 / 2$ of the allocations have associated a high value to individual $i=1$, say $c_{1}=E$, and a low value to individual $i=2$, say $c_{2}=0$ by efficiency. The other $1 / 2$ of the allocations give an equal value to both individuals, say $c_{1}=c_{2}=E / 2$. Therefore, by uniform continuity [and axioms 3 and 4], the mean allocation for individual $i=1$ can be written as,

$$
\phi_{1}^{2}=(E) / 2+(E / 2) / 2=3 E / 4,
$$

and by efficiency $\phi_{2}^{2}=E / 4$, as stated in (3). The same reasoning applies to the case $n=3$, in which $c_{1} \in[E / 3, E), c_{2} \in(0, E / 2)$ and $c_{3} \in(0, E / 3]$, and by efficiency $c_{1}+c_{2}+c_{3}=E$. Therefore, we can think as if $1 / 3$ of the allocations have associated an high value to individual $i=1$, say $c_{1}=E$, and a low value to individuals $i=2,3$, say $c_{2}=c_{3}=0$, by efficiency. Other $1 / 3$ of the allocations have associated a medium value to individuals $i=1,2$, say $c_{1}=c_{2}=E / 2$, and a low value to individual $i=3$, say $c_{3}=0$ by efficiency. The last $1 / 3$ of the allocations give an equal value to all three individuals, say $c_{1}=c_{2}=c_{3}=E / 3$. Again, applying uniform continuity, the mean allocation for individual $i=1$ can be written as,

$$
\phi_{1}^{3}=(E) / 3+(E / 2) / 3+(E / 3) / 3=11 E / 18,
$$


for individual $i=2$ can be written as,

$$
\phi_{2}^{3}=(0 E) / 3+(E / 2) / 3+(E / 3) / 3=5 E / 18,
$$

and by efficiency $\phi_{3}^{3}=2 E / 18$, as stated in (4). For $n=4$, in this case, $c_{1} \in[E / 4, E), c_{2} \in(0, E / 2), c_{3} \in(0, E / 3)$ and $c_{4} \in(0, E / 4]$, and by efficiency $c_{1}+c_{2}+c_{3}+c_{4}=E$. Briefly, the mean allocation for individual $i=1$ is,

$$
\phi_{1}^{4}=(E) / 4+(E / 2) / 4+(E / 3) / 4+(E / 4) / 4=25 E / 48,
$$

for individual $i=2$ is,

$$
\phi_{2}^{4}=(0) / 4+(E / 2) / 4+(E / 3) / 4+(E / 4) / 4=13 E / 48,
$$

for individual $i=3$ is,

$$
\phi_{3}^{4}=(0) / 4+(0) / 4+(E / 3) / 4+(E / 4) / 4=7 E / 48
$$

and by efficiency $\phi_{4}^{4}=3 E / 48$, as stated in (.2). Therefore, for $n$ we have, $c_{1} \in[E / n, E), c_{i} \in(0, E / i)$ for $i \in\{2, \ldots, n-1\}, c_{n} \in(0, E / n]$, and by efficiency $\sum_{i=1}^{n} c_{i}=E$. The mean allocation for individual $i=1$ is $\phi_{1}^{n}=$ $\sum_{k=1}^{n}(E / k) / n$, for individual $i=2$ is $\phi_{2}^{n}=\sum_{k=2}^{n}(E / k) / n$, for individual $i=3$ is $\phi_{3}^{n}=\sum_{i=3}^{n}(E / k) / n$, and so on. Therefore, for general $i$ and $n$ we have (2).

\section{References}

Adams, J. S., 1963. Toward an understanding of inequity-journal of abnormal and social psychology. Washington: American Psychological Association.

Ambec, S., Dinar, A., McKinney, D., 2013. Water sharing agreements sustainable to reduced flows. Journal of Environmental Economics and Management 66 (3), 639-655.

Ambec, S., Ehlers, L., 2008a. Cooperation and equity in the river sharing problem. London: Routledge.

Ambec, S., Ehlers, L., 2008b. Sharing a river among satiable agents. Games and Economic Behavior 64 (1), 35-50.

Ambec, S., Sprumont, Y., 2002. Sharing a river. Journal of Economic Theory 107 (2), 453-462. 
Ansink, E., Weikard, H.-P., 2012. Sequential sharing rules for river sharing problems. Social Choice and Welfare 38 (2), 187-210.

Bahr, G., Requate, T., 2014. Reciprocity and giving in a consecutive three-person dictator game with social interaction. German Economic Review 15 (3), 374-392.

Bonein, A., Serra, D., et al., 2007. Another experimental look at reciprocal behavior: indirect reciprocity. MPRA Paper (3257).

Camerer, C., 2003. Behavioral game theory: Experiments in strategic interaction. Princeton University Press.

Camerer, C., Thaler, R. H., 1995. Anomalies: Ultimatums, dictators and manners. The Journal of Economic Perspectives 9 (2), 209-219.

Carraro, C., Marchiori, C., Sgobbi, A., 2007. Negotiating on water: insights from noncooperative bargaining theory. Environment and Development Economics 12 (02), 329349 .

Charness, G., Sutter, M., 2012. Groups make better self-interested decisions. The Journal of Economic Perspectives 26 (3), 157-176.

Dinar, A., Ratner, A., Yaron, D., 1992. Evaluating cooperative game theory in water resources. Theory and decision $32(1), 1-20$.

Engel, C., 2011. Dictator games: A meta study. Experimental Economics 14 (4), 583-610.

Gengenbach, M. F., WEIKARD, H.-P., Ansink, E., 2010. Cleaning a river: An analysis of voluntary joint action. Natural Resource Modeling 23 (4), 565-590.

Güth, W., Schmittberger, R., Schwarze, B., 1982. An experimental analysis of ultimatum bargaining. Journal of economic behavior \& organization 3 (4), 367-388.

Herings, P. J.-J., Predtetchinski, A., 2012. Sequential share bargaining. International Journal of Game Theory 41 (2), 301-323.

Houba, H., 2008. Computing alternating offers and water prices in bilateral river basin management. International Game Theory Review 10 (03), 257-278.

Kahneman, D., Knetsch, J. L., Thaler, R. H., 1986. Fairness and the assumptions of economics. Journal of business, S285-S300.

Kilgour, D. M., Dinar, A., 2001. Flexible water sharing within an international river basin. Environmental and Resource Economics 18 (1), 43-60.

Littlechild, S. C., Owen, G., 1973. A simple expression for the shapley value in a special case. Management Science 20 (3), 370-372. 
Moulin, H., 2000. Priority rules and other asymmetric rationing methods. Econometrica $68(3), 643-684$.

Parrachino, I., Dinar, A., Patrone, F., 2006. Cooperative game theory and its application to natural, environmental, and water resource issues: 3. application to water resources. Application to Water Resources (November 2006). World Bank Policy Research Working Paper (4074).

Shapley, L. S., 1953. A value for n-person games. In Contributions to the Theory of Games, volume II, by H.W. Kuhn and A.W. Tucker, editors. Annals of Mathematical Studies (28), 307-317.

Thomson, W., 2003. Axiomatic and game-theoretic analysis of bankruptcy and taxation problems: a survey. Mathematical social sciences 45 (3), 249-297.

Thomson, W., 2011. Consistency and its converse: an introduction. Review of Economic Design 15 (4), 257-291.

Thomson, W., Varian, H., 1985. Theories of justice based on symmetry. Chapter 4. In: Hurwicz, L., Schmeidler, D., Sonnenschein, H. (eds.) Social Goals and Social Organizations: Essays in Memory of Elisha Pazner 126.

Thomson, W., et al., 2007. Cost allocation and airport problems. Rochester Center for Economic Research, Working Paper (538).

Wang, Y., 2011. Trading water along a river. Mathematical Social Sciences 61 (2), 124-130. 